\title{
Risk Factors and Clinical Outcomes of Patients with Fluconazole Nonsusceptible Candida tropicalis Bloodstream Infections
}

Pao-Yu Chen ${ }^{1}$, Yu-Chung Chuang ${ }^{1}$, Un-In Wu ${ }^{1}$, Hsin-Yun Sun ${ }^{1}$, Jann-Tay Wang ${ }^{1}$, Wang-Huei Sheng ${ }^{1}$, YeeChun Chen ${ }^{1,2,3}$, Shan-Chwen Chang ${ }^{1,2}$

${ }^{1}$ Department of Internal Medicine, National Taiwan University Hospital, Taipei, Taiwan

2 Department of Medicine, National Taiwan University College of Medicine, Taipei, Taiwan

${ }^{3}$ National Institute of Infectious Diseases and Vaccinology, National Health Research Institutes, Miaoli, Taiwan

\section{Objectives}

Fluconazole non-susceptibility (FNS) rates have increased among $C$. tropicalis isolates in Asia. We aimed to explore the proportions and risk factors of candidemia caused by FNS $C$. tropicalis and the impact of FNS on patient outcomes.

\section{Methods}

There was a prospective Candida bloodstream infections (BSIs) cohort in a single medical center at Northern Taiwan. We retrospectively analyzed the first episode of $C$. tropicalis BSIs between 2011 and 2017. Antifungal susceptibility testing of the first isolate of each episode was performed by the microdilution colorimetric Sensititre YeastOne SYO09 panel, and the results were interpreted according to Clinical and Laboratory Standards Institute (CLSI) species-specific breakpoints.

Results

During the study period, three hundred and forty-four episodes of $C$. tropicalis BSIs were identified. Of them, 58 (16.9\%) first blood isolates were non-susceptible to fluconazole, including 10 isolates belonging to susceptible dose-dependent and 48 isolates belonging to resistance. The proportions of $C$. tropicalis BSIs and fluconazole resistant rates showed an increasing trend by years (both $P=0.07$, Figure 1 ). There were no differences of patient outcomes, including 14-day and 30-day mortality, and persistence, between FNS BSIs and fluconazole susceptible (FS) BSIs (Figure 2). Compared to patients with FS BSIs, those with FNS BSIs were more likely to have moderate to severe liver disease, leukemia, receipt of steroid and antifungal exposure (AE), but less receipt of parental hyperalimentation compared to those infected by FS isolate (all $P<0.05$ ). Of note, around $55 \%$ patients with FNS BSIs were azole naïve (Table 1 ).

\section{Conclusion}

Fluconazole resistance increased in C. tropicalis BSIs, but didn't worsen patient outcomes. Immunocompromised hosts and those with azole exposure were at risk for FNS C. tropicalis BSIs. Further investigations were warranted for azole naïve patients with FNS $C$. tropicalis BSIs.

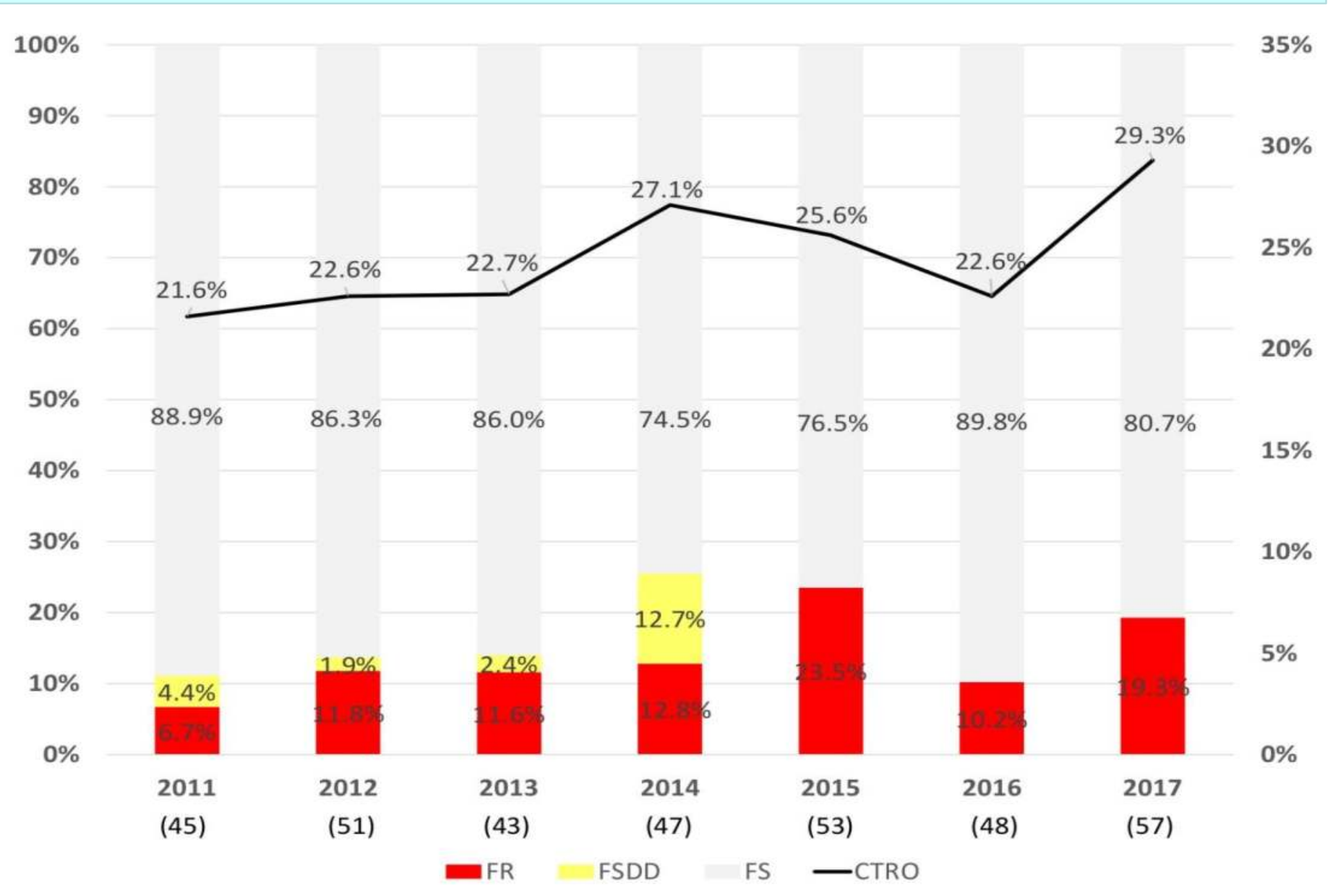

Figure 1. Distributions of fluconazole susceptibility categories among 344 Candida tropicalis blood isolates (primary $Y$ axis) and proportions of $C$. tropicalis in Candida bloodstream infections (secondary $Y$ axis) by year. Primary $Y$ axis: \% of fluconazole resistance (red bar), susceptible dose dependent (yellow bar), and susceptible (grey bar); Secondary $\mathrm{Y}$ axis: $\%$ of $C$. tropicalis (black line); $\mathrm{X}$ axis: Year; Numbers below year: numbers of isolates in a given year

Copyright @ 2018 Pao-Yu Chen; Contact: chenpaoyu@gmail.com

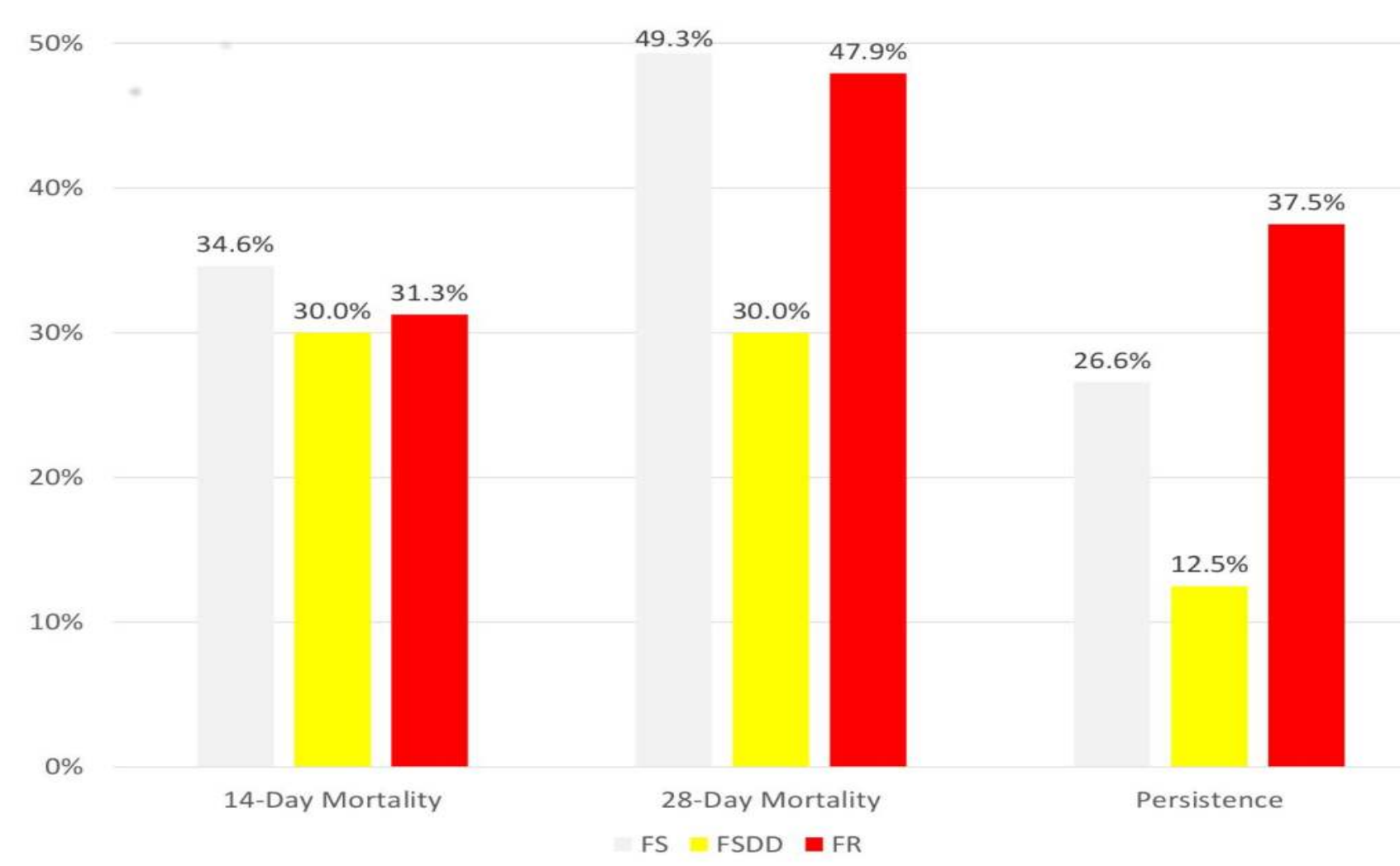

Figure 2. Comparisons of 14-day and 28-day mortality and persistent BSI patients grouped by fluconazole susceptibility categories. $Y$ axis: $\%$ of patients with above outcomes. $X$ axis: Three different outcomes. Fluconazole resistance ( $F R$, red bar), susceptible dose dependent (FSDD, yellow bar), and susceptible (FS, grey bar); persistence defined as $C$. tropicalis isolated from blood at least 5 days after onset

Table 1. Comparisons of clinical characteristics between FS and FNS $\boldsymbol{C}$. tropicalis BSIs.

\begin{tabular}{|c|c|c|}
\hline Characteristics" & $\begin{array}{c}\text { FS Group } \\
(n=286)\end{array}$ & $\begin{array}{c}\text { FNS Group } \\
(n=58)\end{array}$ \\
\hline Age, years & $62.4(53.0-74.4)$ & $63.4(55.1-72.1)$ \\
\hline Male & $165(57.7)$ & $36(62.1)$ \\
\hline Charlson comorbidity index & $3.5(2.0-6.0)$ & $4.0(2.0-7.0)$ \\
\hline Congestive heart failure & $25(8.7)$ & $4(6.9)$ \\
\hline Mild liver diseases & $45(15.7)$ & $6(10.3)$ \\
\hline $\begin{array}{l}\text { Moderate-to-severe liver } \\
\text { diseases }^{\$^{*}}\end{array}$ & $13(4.5)$ & $6(10.3)$ \\
\hline Moderate-to-severe renal diseases & $83(29.0)$ & $13(28.3)$ \\
\hline Diabetes mellitus & $63(22.0)$ & $18(31.0)$ \\
\hline Solid tumor & $151(52.8)$ & $27(46.6)$ \\
\hline Leukemia\$ & $38(13.3)$ & $13(22.4)$ \\
\hline Lymphoma & $18(6.3)$ & $3(5.2)$ \\
\hline Parental hyperalimentation $\$$ & $39(84.8)$ & $29(63.0)$ \\
\hline Steroid use & $133(46.5)$ & $37(63.8)$ \\
\hline Chemotherapy & $123(43.0)$ & 30 (51.7) \\
\hline Neutropenia & $155(54.2)$ & 34 (58.6) \\
\hline Antifungal exposure $\$ *$ & $34(11.9)$ & $26(44.8)$ \\
\hline ICU onset & 85 (29.9) & $20(34.5)$ \\
\hline APACHE II score & $20.0(15.0-26.0)$ & $19.0(15.25-26.0)$ \\
\hline Primary BSI & $122(42.7)$ & $23(39.7)$ \\
\hline Catheter-related BSI & $111(38.8)$ & $18(31.0)$ \\
\hline Urinary tract infection & $51(17.8)$ & $13(22.4)$ \\
\hline Intra-abdomen infection & $10(3.5)$ & $2(3.4)$ \\
\hline $\begin{array}{l}\text { Appropriate antifungal agents } \\
\mathrm{w} / \text { in } 48 \mathrm{hr} \$\end{array}$ & $244(85.3)$ & $17(29.3)$ \\
\hline $\begin{array}{l}\text { Removal of central venous } \\
\text { catheters } w / \text { in } 48 \mathrm{hr}\end{array}$ & $131(55.0)$ & 31 (64.6) \\
\hline
\end{tabular}

\# Continuous variables: median (IQR); Categorical variables: numbers (\%)

$\$$ Univariate analysis, $P<0.05$

* Multivariate logistic regression: Antifungal exposure (aOR, 6.00; 95\% Cl, 3.17-11.36); Moderate-to-severe liver diseases (aOR, 3.14; 95\% $\mathrm{Cl}, 1.05-9.33$ ) 